\title{
Références bibliographiques du dossier : « Diplômes et examens de l'enseignement secondaire »
}

Prunelle Charvet

\section{CpenEdition}

Journals

Édition électronique

URL : https://journals.openedition.org/ries/1445

DOI : $10.4000 /$ ries. 1445

ISSN : 2261-4265

Éditeur

France Education international

Édition imprimée

Date de publication : 1 décembre 2004

Pagination : 87-96

ISBN : 978-2-85420-564-0

ISSN : $1254-4590$

Référence électronique

Prunelle Charvet, «Références bibliographiques du dossier : "Diplômes et examens de l'enseignement secondaire » », Revue internationale d'éducation de Sèvres [En ligne], 37 | décembre 2004, mis en ligne le 18 novembre 2011, consulté le 05 juillet 2021. URL : http://journals.openedition.org/ries/1445 ; DOI : https://doi.org/10.4000/ries.1445 


\section{Références bibliographiques}

\section{Prunelle Charvet}

Cette bibliographie, non exhaustive, recense des documents ayant comme champ d'investigation les modalités et les fonctions des examens, contrôles et validation des connaissances dans le second degré. Après des études transnationales sur les certifications de l'enseignement secondaire, elle propose des références bibliographiques traitant de la reconnaissance des diplômes en Europe. Enfin, des ouvrages présentant plus particulièrement les dispositifs français pour l'enseignement général et l'enseignement technique sont cités. Les documents faisant référence à la reconnaissance des diplômes en Europe sont postérieurs à 1992, date de la signature du traité de Maastricht sur l'Union européenne qui, reconnaissant notamment le droit de circuler et de résider librement dans les pays de la Communauté, a constitué une nouvelle étape dans la réflexion menée à ce sujet.

La majorité des documents mentionnés sont consultables au centre de ressources du Centre international d'études pédagogiques ou sont en ligne sur Internet. Bibliographie arrêtée le 30 septembre 2004.

\section{Les systèmes de certification : études transnationales}

BAKKER Steven, WOLF Alison, Upper secondary examinations and entry to university : the school-university transition in an age of mass higher education, Assessment in Education : Principles, Policy \& Practice, 2001, Vol. 8, no 3, pp. 285-408

Cet article soulève différentes questions liées à la massification de l'éducation dans les pays de l'OCDE telles que l'influence des développements de l'éducation sur les examens et les tests d'entrée à l'université et les facteurs qui influent sur la nature des examens de premier cycle de l'enseignement secondaire.

BERTRAND Olivier réd., DURAND-DROUHIN Marianne dir. OCDE: Organisation de coopération et de développement économiques/Paris, Qualifications et compétences professionnelles dans l'enseignement technique et la formation professionnelle : évaluation et certification, OCDE/Paris, 1996, 128 p.

Ce volume est la synthèse des travaux réalisés lors du séminaire de Porto (octobre 1992), dans le cadre du projet sur «le rôle nouveau de l'enseignement technique et la formation professionnelle (VOTEC)» lancé par l'OCDE. La première partie contient des analyses à caractère général et aborde aussi les thèmes suivants : aspects pédagogiques de la certification, reconnaissance et transférabilité des qualifications, problèmes institutionnels posés. La deuxième partie est constituée d'études par pays permettant de se référer à trois approches institutionnelles et culturelles de la formation professionnelle. 
CAVDAR Tomris, Analyse comparative des systèmes de contrôle des connaissances, d'évaluation et de certification français, allemand et turc, Formation professionnelle, avril 2001, $n^{\circ} 22$, p. 51-58

Cet article présente une analyse comparative des systèmes de contrôle des connaissances, d'évaluation et de certification français, allemand et turc.

Commission Européenne, Eurostat : office statistique de Bruxelles, Eurydice : réseau européen d'information sur l'éducation, Les chiffres clés de l'éducation en Europe 2002, Office des publications officielles des Communautés européennes/ Luxembourg, 2002, 262 p., annexes, (Les chiffres clés de l'éducation en Europe) L'édition 2002 de cet ouvrage couvre trente pays européens. Résultat d'une collaboration entre Eurydice, réseau d'information sur l'éducation en Europe et Eurostat, office statistique de l'Union européenne, ce rapport a pour objectif de mettre en évidence la diversité, les similitudes et les tendances d'évolution des systèmes éducatifs de ces pays à l'aide d'une série de 145 indicateurs. Dans le chapitre consacré à l'enseignement secondaire, une partie traite de la certification.

\section{ECKSTEIN Max A., NOAH Harold J., Examinations : comparative and inter-} national studies, Pergamon Press/Oxford, 1992, 179 p., index, (Pergamon comparative and international education series)

Dans une perspective comparative, l'ouvrage offre un aperçu des systèmes d'examens du Japon, de la Chine, de l'Europe de l'Ouest, des Etats-Unis et des pays du tiers monde. Il analyse notamment les fonctions des examens de sortie de l'enseignement secondaire dans les sociétés respectives de chacun de ces pays.

\section{EURYDICE : Réseau d'information sur l'éducation en Europe, Glossaire européen de l'éducation. Volume 1 - Deuxième édition. Examens, diplômes et titres, EURYDICE/Bruxelles, 2004, 268 p., (Repères et références) \\ Ce glossaire présente environ mille titres spécifiques utilisés dans les systèmes éducatifs de trente pays européens en matière d'examens, de diplômes et de titres et, ce faisant, constitue une compilation de la terminologie employée dans le domaine de la certification. Le docu- ment concerne uniquement les examens ou les certifications des formations dispensés dans les établissements d'enseignement ou organisés en partenariat avec eux et seuls figurent les diplômes et titres académiques à part entière. La première partie contient la liste des termes classés par ordre alphabétique, la seconde partie propose des tableaux récapitulatifs par pays. Ce document est aussi disponible sur Internet (http://www.eurydice.org).}

\section{FIALAIRE Jacques, L'évolution des diplômes de fin d'études secondaires dans} les pays de l'Union européenne et la question de la réforme du baccalauréat, Savoir éducation formation, mars 1998, $n^{\circ} 1$, p. 97-109

En comparant les régimes de diplômes de fin d'études secondaires dans les quinze pays de l'Union européenne, l'auteur met en évidence la diversité de ces diplômes étroitement liée à l'originalité de leurs systèmes éducatifs respectifs. Cependant, des tendances communes marquent l'évolution des régimes des diplômes de fin d'études secondaires.

\section{GOLDSTEIN H., Educational standards, Oxford University Press/Oxford/} Royaume Uni, 2000, vii-158 p., bibliogr.

Cet ouvrage est une étude comparative sur ce que sont les niveaux, une notion étroitement liée au contexte historique et social de chaque pays. Les auteurs considèrent qu'il est difficile, 
voire impossible, de définir objectivement les niveaux en éducation. Ils analysent le rôle des systèmes publics d'examens et les différents moyens qui permettent de mesurer les niveaux.

OCDE : Organisation de coopération et de développement économiques. CERI : Centre pour la recherche et l'innovation dans l'enseignement/Paris, Regards sur l'éducation 2004 : les indicateurs de l'OCDE, OCDE/Paris, 492 p., annexes, glossaire, statistiques

L'édition 2004 de Regards sur l'éducation insiste sur la qualité des résultats de l'éducation, sur les leviers politiques et les facteurs contextuels qui les conditionnent, ainsi que sur les diverses formes de rendement privé et social générées par les investissements dans l'éducation. Le premier chapitre examine notamment les taux d'obtention d'un diplôme de deuxième cycle de l'enseignement secondaire et du niveau tertiaire. Pour la première fois, des indicateurs sur l'évolution du rendement de l'éducation sur le marché du travail sont fournis.

PERKER Henriette coord., BECCARELLI Catherine coord., Se former en Europe : dossier, Actualité de la formation permanente, septembre 2001, $n^{\circ} 173$, p. 31-145 Ce dossier souligne le rôle essentiel de la formation dans l'Europe de demain. Ses nombreuses contributions permettent de mieux appréhender les évolutions en cours marquées essentiellement par la mobilité et la reconnaissance des qualifications et de l'expérience professionnelle. La dernière partie du dossier rassemble des présentations synthétiques du système de formation initiale, professionnelle et continue dans quatorze États membres de l'Union européenne.

VERDIER Eric coord., Construction et négociation des diplômes : numéro spécial, Formation emploi, décembre 1995, $n^{\circ}$ 52, p. 3-188, bibliogr.

Cet article de base passe en revue les différentes théories économiques du diplôme et aborde notamment le processus de création et d'élaboration des diplômes professionnels et technologiques. Une bibliographie sur la genèse des diplômes complète ce numéro spécial.

VINCENS Jean, Dynamique de l'éducation et systèmes éducatifs, Formation professionnelle, avril 2002, $n^{\circ} 25$, p. 9-26

Cet article s'appuie sur une recherche collective, le projet EDEX (Educational Expansion and Labour Market), financé par la Communauté Européenne. L'auteur examine les modalités et les conséquences de l'augmentation massive du nombre d'actifs diplômés dans cinq pays européens : Allemagne, Espagne, France, Italie et Royaume-Uni au cours des quarante dernières années.

\section{La reconnaissance des diplômes en Europe}

EURYDICE : Réseau d'information sur l'éducation dans l'Union européenne et les pays de l'AELE-EEE, LAFONTAINE Annette coord., LAFONTAINE Dominique coord., et al., Les conditions d'accès à l'enseignement supérieur dans la Communauté européenne, EURYDICE/Bruxelles, 1993, 71 p.

Le document informe sur les conditions d'accès à l'enseignement supérieur dans la Communauté européenne, les exigences linguistiques, les formalités d'inscription et de séjour, et notamment la reconnaissance des diplômes. Il est constitué de deux parties : le premier 
volet est une comparaison par thème, le second volet traite le sujet sous forme de fiches synthétiques par pays.

FRAZIER Carole, L'éducation et la Communauté européenne, CNRS/Paris, 1995, $338 p$.

La prise de conscience des liens entre les systèmes de formation et le champ économique, dans une société en constante mutation, impose de s'interroger sur la compétence de la Communauté européenne en matière d'éducation. L'étude, issue d'une thèse, envisage la question à travers l'aspect juridique. L'auteur expose l'évolution de l'approche communautaire dans une première partie consacrée à l'application du principe d'égalité de traitement et de la reconnaissance mutuelle des diplômes; une seconde partie décrit les interventions communautaires, illustrées par les programmes et la mise en ouvre d'une politique dans un domaine qui est le terrain privilégié du principe de subsidiarité.

PERTEK Jacques, La reconnaissance des diplômes en Europe, PUF/Paris, 1999, 128 p., bibliogr., (Que sais-je?)

Après avoir fait le point sur la diversité des dispositifs dont dépendent la pratique d'une profession et la poursuite d'études dans un autre État de la Communauté, l'auteur explique la dynamique de la reconnaissance vers laquelle la Communauté européenne tend. Il s'interroge ensuite sur la reconnaissance plus spécifique des diplômes dans la grande Europe et propose comme élément de réponse, d'utiliser de manière plus effective le droit à la libre circulation reconnu aux citoyens européens, la réalisation d'un espace européen des libertés, de l'éducation et des idées. Des extraits du rapport du Groupe de haut niveau sur la libre circulation des personnes (1997) figurent en annexe.

PERTEK Jacques dir., La reconnaissance des qualifications dans un espace européen des formations et des professions, Bruylant/Bruxelles, 1998, 370 p.

Cette ouvrage présente le compte rendu des travaux du séminaire organisé sous le même titre à la Faculté de Droit de l'Université Lille II, en coopération entre le ministère de l'Éducation nationale (France), la Fondation pour les Études européennes (Pays-Bas) et l'académie de droit européen (ERA) de Trèves (RFA), les 29, 30 et 31 mai 1997. Il s'agit d'une réflexion sur le droit à la libre circulation professionnelle des citoyens à l'intérieur de la Communauté et sur la reconnaissance des diplômes et des qualifications, celle-ci étant nécessaire pour réaliser cette mobilité.

PERTEK Jacques, L'Europe des diplômes et des professions, Bruylant/Bruxelles, 1998, 286 p., bibliogr.

L'ouvrage traite de l'action de la Communauté européenne en matière d'éducation et de formation professionnelle qui s'est intensifiée progressivement. Elle porte sur la reconnaissance mutuelle des diplômes à des fins professionnelles, organisée par des directives communautaires, ainsi que sur l'accès à l'enseignement supérieur dans un autre Etat et la coopération entre établissements d'enseignement. Les programmes communautaires cherchent à encourager la mobilité des enseignants et des jeunes en formation et à favoriser la libre circulation. Les progrès réalisés dans ce sens doivent beaucoup à la jurisprudence de la Cour de justice du Luxembourg, dont les principaux arrêts dans ce domaine figurent en annexe. 


\title{
Les systèmes de certification français
}

\section{Les certifications d’enseignement général}

\begin{abstract}
BEAUD Stéphane, $80 \%$ au bac et après? Les enfants de la démocratisation scolaire, La Découverte/Paris, 2003, $n^{\circ}$ 155, 342 p. (Textes à l'appui/enquête de terrain)

Cet ouvrage, résultat d'une enquête de dix années, relate les illusions et désillusions des «enfants de la démocratisation scolaire», engagés dans la voie des études longues. L'auteur met en lumière l'ambivalence de la politique volontariste de démocratisation scolaire.
\end{abstract}

BEAUD Stéphane, Le baccalauréat : passeport ou mirage?, Problèmes politiques et sociaux, 2003, $n^{\circ} 891,120 \mathrm{p}$.

Après avoir fait le point sur les différentes politiques éducatives relatives au baccalauréat et leurs effets sur l'enseignement professionnel, l'ouvrage retrace le rôle des principaux acteurs du système éducatif concernés et propose un bilan statistique. Il met en évidence les différentes inégalités scolaires mesurées par rapport à ce diplôme et les études post-bac. Enfin, il analyse la corrélation entre la certification scolaire et l'insertion professionnelle et propose des perspectives sur l'avenir du diplôme.

BELHOSTE Bruno dir., CASPARD Pierre, TOUSSAINT Daniel, et al., L'examen : évaluer sélectionner, certifier $X V I^{e}-X X^{e}$ siècles, Histoire de l'éducation, mai 2002, $n^{\circ} 94,230 p$.

Après avoir posé quelques pistes de réflexion sur le thème de l'examen scolaire, ce numéro retrace l'historique et décrit l'organisation de différents types d'examens : certificat de fin d'études, brevet de capacité de l'instruction primaire, baccalauréat, concours d'admission à l'École polytechnique. Il traite également des origines de la «science des examens» et des conditions de collecte et de conservation des copies d'examen.

BELLOUBET-FRIER Nicole, 30 propositions sur l'avenir du lycée, mars 2002, 76 p. téléchargées du site : www.education.gouv.fr

À la suite des recommandations de J. Lang, alors ministre de l'Éducation nationale, l'auteur centre sa réflexion sur les points suivants - analyse des raisons de la stagnation, voire de la diminution du taux d'accès d'une génération de lycéens au bacalauréat et volonté d'accroître la proportion de bachelier - propositions visant à recentrer les missions de l'enseignement secondaire aussi bien dans la voie générale que technologique ou professionnelle - redéfinition des processus d'orientation au collège, de la liaison collège/lycée et lycée/études supérieures.

BLOCH Daniel, CHAMONARD D., BOULENC J. et al., Les bacheliers "avec mention" et leurs poursuites d'études, Education \& Formations, septembre 2001, $n^{\circ}$ 60, pp. 71-74

À partir de données statistiques, l'article décrit le profil des bacheliers ayant obtenu leur baccalauréat avec mention selon plusieurs critères : origines sociales, poursuites d'études... 
BRIFFAUX Amélie, Résultats définitifs de la session 2003 du baccalauréat, Note d'information - DEP, mars 2004, $n^{\circ}$ 04.07, 6 p. téléchargées du site : http://www. education.gouv.fr

Cette note offre un éclairage statistique sur l'obtention du baccalauréat selon les filières, le sexe et la génération ainsi que sur les bacheliers admis avec mention.

CLAVAL Bruno, SABOULIN Michel de, Le diplôme d'accès aux études universitaires Note d'information - DEP, juin 2000, $n^{\circ} 00.17,5$.

Cette note d'information présente de manière synthétique le fonctionnement du DAEU (diplôme d'accès aux études universitaires) et en offre un aperçu historique à travers les différents dispositifs qui l'ont précédé. Elle met en évidence les principales tendances qui ressortent de l'analyse des statistiques : les options choisies par les candidats, la proportion d'hommes et de femmes inscrits...

FERRAIT Laure, KLEINHOLT Sylvie, Résultats définitifs du diplôme national du brevet (public et privé) session 2003, Note d'information - DEP, avril 2004, $n^{\circ}$ 04.10, 6 p. téléchargées du site : http://www.education.gouv.fr

Ce document commente les statistiques des résultats définitifs du diplôme national du brevet (public et privé) en 2003. Un très bref aperçu historique de ce diplôme figure à la fin du document.

LELIEVRE Claude, Les politiques scolaires mises en examen : douze questions en débat, ESF/Paris, 2002, 208 p. (Pédagogies essais)

L'auteur a sélectionné douze questions relatives au système éducatif français qui faisaient débat au printemps 2002. Il fait un bref historique des interventions des hommes politiques les plus connus dans ces domaines sensibles depuis 1958. Outre des thèmes tels que la violence à l'école, les rythmes scolaires ou encore la réforme du collège, une partie est consacrée à la question du baccalauréat et de l'entrée à l'université.

LEMAIRE Sylvie, Que deviennent les bacheliers après leur baccalauréat? Évolutions 1996-2002, Note d'information - DEP, juin 2004, nº4.14, 6 p. téléchargées du site : http://www.education.gouv.frl

Ce document présente les conclusions tirées après analyse des statistiques sur l'évolution de l'orientation des bacheliers à l'entrée dans le supérieur entre 1996 et 2002.

Ministère de la Jeunesse, de l'Éducation, et de la recherche. Haut Conseil de l'évaluation de l'école, Avis du Haut conseil de l'évaluation de l'école. Apprécier et certifier les acquis des élèves en fin de collège : diplôme et évaluationsbilans, Avis du HCEE $n^{\circ}$ 2, juin 2001, 4 p. téléchargées du site: http://cisad.adc. education.fr/hcee

Cet avis insiste sur la nécessité de reconstruire une certification de fin de scolarité obligatoire à la fin du collège. Il souligne l'importance des évaluations-bilans. Apprécier et certifier les acquis des élèves constitue en effet un double enjeu de politique éducative. Il s'agit de vérifier que le système permet aux élèves d'accéder à la maîtrise des savoirs, mais aussi d'évaluer l'acquisition des compétences de chacun. 
Ministère de l'Éducation nationale. Délégation aux relations internationales et à la coopération/Paris, Enseignement scolaire et dimension internationale dispositifs et acteurs en France et à l'étranger, Ministère de l'Education Nationale, mai 2002, n.p. (Dossiers spéciaux)

Ce dossier présente d'une part, les dispositifs de l'enseignement scolaire en France (les sections européennes et les sections internationales) et d'autre part, ceux mis en place à l'étranger (les établissements gérés par l'AEFE et les écoles européennes). Il traite ensuite des partenariats et de la coopération (sections bilingues, programmes ELCO, coopération franco-allemande...). La dernière partie consacrée à l'évaluation des compétences aborde les questions de l'indication section européenne, section orientale sur le diplôme du baccalauréat, l'option internationale du baccalauréat, le baccalauréat franco-allemand, l'ABIBAC (ABItur/BACcalauréat), les certifications étrangères.... Le dossier s'achève sur deux pages de glossaire.

Ministère de la Jeunesse, de l'Éducation nationale et de la Recherche. DPD : Direction de la programmation et du développement, L'état de l'école $n^{\circ} 13$ : 30 indicateurs sur le système éducatif français, Ministère Jeunesse Éducation Recherche/Paris, 2003, vol. 13, 77 p. (L'état de l'école)

Comme dans les précédentes éditions, cette treizième livraison de «l'État de l'école» tente de répondre, grâce à un ensemble de statistiques, à un certain nombre de questions sur le système éducatif français. Trois chapitres s'intéressent respectivement à l'emploi et au devenir professionnel des sortants du second degré, aux accès au niveau IV et $\mathrm{V}$ de formation et enfin, aux bacheliers et à leur accès immédiat dans le supérieur.

Ministère de la Jeunesse, de l'Éducation nationale et de la Recherche. DEP : Direction de l'évaluation et de la prospective/Paris, PERETTI Claudine, Repères et références statistiques sur les enseignements, la formation et la recherche : 2004, Ministère de l'Éducation Nationale. DPD/Direction de la programmation, 2004, 366 p. (RERS)

Cette collection, propose d'année en année, une information suivie et actualisée sur tous les aspects structurels de l'éducation et de la recherche. Un chapitre entier est consacré aux examens et sorties. Il présente des données statistiques sur le diplôme national du brevet, les diplômes de l'enseignement technologique et professionnel, la réussite au baccalauréat selon la série, le sexe, l'âge, la catégorie sociale et la génération. Sont abordées ensuite la réussite des bacheliers et non bacheliers au DEUG et au DUT et l'insertion professionnelle des jeunes à la sortie du lycée.

SOLAUX Georges, Le baccalauréat, CNDP/Paris, Documentation française/Paris, 1995, 152 p., bibliogr. (Systèmes éducatifs)

À travers ses six chapitres, cet ouvrage aborde successivement la dynamique du diplôme dans une perspective historique, la genèse de la rénovation pédagogique des lycées, la récente réforme du baccalauréat, la diversité des baccalauréats en France, les dits et les non-dits sur cet examen et termine par quelques comparaisons internationales.

\footnotetext{
TOULEMONDE Bernard dir., Le système éducatif en France, Documentation française/Paris, 2003, 191 p. (Les notices)

L'ouvrage fournit une présentation claire et précise de l'ensemble du système éducatif français : les fondements, l'organisation administrative, l'organisation des enseignements et les enjeux. Un chapitre est notamment consacré aux diplômes, aux qualifications et à l'insertion professionnelle (relation formation/emploi). La question de l'ouverture internationale et européenne du système éducatif français est également abordée.
} 


\section{Les certifications de l'enseignement technique}

AFPA : Association nationale pour la formation professionnelle des adultes, Recueil de textes officiels : vocabulaire $n^{\circ}$ spécial : certification, AFPA/ Montreuil/France, mars 2003, $n^{\circ}$ spécial, 78 p.

Ensemble des textes - lois, décrets, arrêtés et circulaires - portant sur la certification professionnelle : délivrance de diplôme, congé pour validation des acquis, informations sur la validation des acquis de l'expérience (VAE). Dans une deuxième partie, un lexique permet de faire le point sur l'ensemble du vocabulaire qui concerne la certification.

CEREQ : Centre d'études et de recherches sur les qualifications, Ministère de l'Éducation nationale. Direction de l'enseignement scolaire/Paris, La construction de la certification : quelles méthodes pour quels usages? : journées de travail DESCO-CEREQ - Paris 13 et 14 septembre 2001, CEREQ/Marseille/France, 2002, $n^{\circ} 161,151$ p. (Document)

Les 13 et 14 septembre 2001, un séminaire a été organisé pour faire le point sur les usages des référentiels de diplôme. Il a réuni certains concepteurs de la certification : l'inspection générale de l'Éducation nationale, la direction de l'Enseignement supérieur, et des chercheurs. Cet ouvrage rassemble l'ensemble des conférences, tables rondes et ateliers. Après avoir fait une analyse critique de la nature et des usages des référentiels, les interventions ont porté sur les questions ouvertes par le développement de la validation des acquis professionnels (VAP) et validation des acquis de l'expérience (VAE), nouvelle voie d'accès aux diplômes. La construction des certifications est posée comme un enjeu socio-économique et non plus seulement pédagogique et renvoie à la pertinence des diplômes existants et à créer.

COUPPIÉ Thomas, LOPEZ Alberto, Quelle utilité les CAP et BEP tertiaires ontils aujourd'hui ?, Bref, avril 2003, $n^{\circ} 196,4$ p. téléchargées du site : http://www.cereq.fr Ce document met en évidence les difficultés rencontrées par les jeunes titulaires de CAP et de BEP tertiaires face à la concurrence des plus diplômés. Il interroge sur la valeur de ces diplômes sur le marché du travail et fait le point sur les familles d'emplois qui leur sont ouvertes ou fermées.

FORESTIER Christian, Ministère de l'Éducation nationale, de la Recherche et de la Technologie, Propositions pour une rénovation de la voie technologique, Rapport, 1999, 68 p. téléchargées du site : http://www.ladocumentationfrancaise.fr/brp

Après un historique de la création de la voie technologique, ce rapport présente les différentes filières qui existent. En vue de la rénovation de cette filière, plusieurs pistes de réflexion sont proposées : élargir le champ des baccalauréats scientifiques et freiner le développement des formations tertiaires, préserver le palier d'orientation de fin de seconde, mettre en place un nombre limité de baccalauréats technologiques (préparant à toutes les formations supérieures) et renforcer le caractère spécifique de la pédagogie.

Haut Comité éducation-économie-emploi, GAURON André, LABRUYERE Chantal et al., La transition professionnelle des jeunes sortant de l'enseignement secondaire : rapport d'activité 2001-2002, Documentation française/Paris, 2002, 199 p., bibliogr.

Ce rapport décrit le contexte du système éducatif français de ces vingt dernières années, analyse les conditions d'insertion des jeunes et tente d'apprécier les tensions des dix prochaines années dans le milieu éducatif. Il présente quelques politiques en matière d'inser- 
tion et de formation professionnelles ainsi que des recommandations et des propositions autour de l'objectif de formation et le développement tout au long de la vie.

\section{IGEN : Inspection générale de l'Éducation nationale, Évaluation du contrôle en cours de formation dans l'enseignement professionnel. Rapport, août 1999, 19 p. téléchargées du site : www.education.gouv.fr}

Après un bref rappelle de l'historique du CCF (Contrôle en Cours de Formation), ce rapport met en évidence les raisons pour lesquelles cette innovation majeure a trop longtemps fait l'objet d'un flou conceptuel. Il insiste sur la disparité des textes d'application et des référentiels de diplômes. Il aborde ensuite le comportement des acteurs (professeurs, chefs d'établissement, élèves, tuteurs en entreprise, inspecteurs...) sur le terrain et fait quelques propositions visant un développement harmonieux du CCF.

\section{MAILLARD Fabienne, Les référentiels des diplômes professionnels confrontés à} leurs critiques : une mise en valeur de leurs enjeux sociaux, Revue française de pédagogie, décembre 2003, $n^{\circ} 145$, p. 63-76

Cet article analyse les principales critiques qu'adressent les chercheurs et les acteurs de la formation aux référentiels des diplômes professionnels. Il insiste sur les problèmes que soulève la standardisation des référentiels d'activités professionnelles et des référentiels de certification.

Ministère de l'Éducation nationale, de la Recherche et de la Technologie, Rénovation des diplômes professionnels, Bulletin officiel de l'Education nationale, octobre 2003, hors-série $n^{\circ} 10$, vol. 28 et 29, p. 2309-2460

ONISEP : Office national d'information sur les enseignements et les professions, Les BEP : brevets d'études professionnelles, Onisep/Marne-la-Vallée, 2003, 135 p., (Diplômes)

L'ouvrage présente les objectifs, les débouchés, les profils, les programmes et les examens de ce diplôme national décliné en cinquante spécialités et sa double finalité : insertion professionnelle et poursuite d'études.

ONISEP : Office national d'information sur les enseignements et les professions, Entrer en bac professionnel : les conditions d'accès, Onisep/Marne-la-Vallée, 2002, 60 p. (Diplômes)

Ce document fait le point, pour chaque baccalauréat professionnel, sur les conditions d'admission et les diplômes prioritaires nécessaires.

\section{PERKER Henriette, MARTEL Dorian, Les certificats de qualification profes-} sionnelle, Centre INFFO/Paris-La Défense, 2001, 124 p. (Guide technique)

Entre 1987 et 2001, plus de 400 certificats de qualification professionnelle ont été créés par les partenaires sociaux de 30 branches professionnelles et une vingtaine d'autres branches ont signé des accords prévoyant leur mise en œuvre. La troisième édition de ce guide apporte les informations les plus récentes sur les accords signés et les pratiques. Les auteurs décrivent l'origine et le contexte de la création de ces certificats, leur organisation, le public à qui ils s'adressent et comment ils se situent par rapport aux autres certifications. 
VUILLET Claudie, SICILIANO Dominique, Qu'évalue-t-on avec les épreuves du baccalauréat professionnel?, $n^{\circ} 10$, novembre 2003, 56 p. téléchargées du site: http://cisad.adc.education.fr/hcee

Après avoir retracé l'évolution du baccalauréat professionnel, ce rapport fait le point sur les compétences et les connaissances qui sont évaluées par les épreuves de ce diplôme. Ce dernier est ensuite examiné au regard de sa double finalité : l'insertion professionnelle ou la poursuite d'études. 